J. Austral. Math. Soc. (Series A) 26 (1978), 501-510

\title{
NECESSARY AND SUFFICIENT CONDITIONS FOR THE EXISTENCE OF A GENERALIZED STIELTJES INTEGRAL
}

\author{
A. M. RUSSELL
}

(Received 9 May 1977)

Communicated by A. P. Robertson

\begin{abstract}
In Russell (1973) a Riemann-type necessary and sufficient condition was given for the existence of $\int_{a}^{b} f\left(d^{k} g / d x^{k-1}\right.$ ) (defined also in Russell (1975)) when $f$ was bounded and $g$ was $k$-convex in $\left[a^{\prime}, b^{\prime}\right]$. In this paper we present necessary and sufficient conditions for the existence of a particular Stieltjes-type integral without imposing a convexity condition upon $g$. These conditions are used to obtain an additivity result for the integral over adjoining intervals without any additional restrictions being imposed upon the functions involved.
\end{abstract}

Subject classification (Amer. Math. Soc. (MOS) 1970): 26 A 42.

\section{Introduction}

We shall follow the notation of Hildebrandt (1963), and speak of norm-integrals and $\sigma$-integrals, the first being defined as a limit when the maximum length of subintervals of a subdivision tends to zero, while the latter is defined as a limit under successive refinements of a subdivision. We shall accordingly prefix the integrals with the symbols ' $N$ ' or ' $\sigma$ '.

It is well known in the theory of Riemann-Stieltjes integration that the additive property

$$
\text { (N) } \int_{a}^{b} f d g=(N) \int_{a}^{c} f d g+(N) \int_{c}^{b} f d g,
$$

where $a<c<b$, does not hold unconditionally. For example, if both integrais on the right-hand side of (1) exist, the integral on the left does not exist if $f$ and $g$ have a common discontinuity at $c$. Such restrictions can be removed by considering 
$\sigma$-integrals in which case the additivity result (1) is free of constraints and can be stated: 'If any two of the integrals $(\sigma) \int_{a}^{b} f d g,(\sigma) \int_{a}^{c} f d g,(\sigma) \int_{c}^{b} f d g$ exist, so does the third, and

$$
(\sigma) \int_{a}^{b} f d g=(\sigma) \int_{a}^{c} f d g+(\sigma) \int_{c}^{b} f d g
$$

In Russell (1973) an analogue of (1) was established, but again restrictions upon the behaviour of the functions $f$ and $g$ in a neighbourhood of $c$ had to be imposed in order to achieve 'additivity'. The integrals involved were norm-integrals. Even if second-order $\sigma$-integrals are introduced, additivity is not achieved. This is illustrated by the following example: If

$$
f(x)=1 \text { and } g(x)=|x| \text { for all } x,
$$

then

$$
(\sigma) \int_{-1}^{1} f \frac{d^{2} g}{d x}=(\sigma) \int_{-1}^{0} f \frac{d^{2} g}{d x}=(\sigma) \int_{0}^{1} f \frac{d^{2} g}{d x}=2
$$

If we introduce a slightly modified second-order $\sigma$-integral, an additivity result can be obtained without imposing extra restrictions upon $f$ and $g$. To achieve this somewhat more desirable result we first obtain necessary and sufficient conditions for the existence of the integral, denoted by $(\sigma) \int_{a}^{b} f\left(d^{2} g / d x\right)$, and show that they exclude the possibility of $f$ being discontinuous and $g$ non-differentiable at the same point.

\section{Notation and preliminaries}

As mentioned previously we will be concerned with a second-order RiemannStieltjes $\sigma$-integral. In order to define such an integral we need a particular type of subdivision of the closed interval $[a, b]$.

Definition 1. $\Gamma$ subdivisions. We will denote by $\Gamma\left(x_{-1}, x_{0}, \ldots, x_{n}, x_{n+1}\right)$, or often more briefly by $\Gamma$, a subdivision of the interval $[a, b]$ of the form

$$
a^{\prime} \leqslant x_{-1}<a=x_{0}<x_{1}<\ldots<x_{n}=b<x_{n+1} \leqslant b^{\prime},
$$

where $a^{\prime}$ and $b^{\prime}$ are fixed, and $a^{\prime}<a<b<b^{\prime}$. For convenience, such a set of points will be called a $\Gamma$ subdivision of $[a, b]$, even though it is not strictly a subdivision of $[a, b]$. 
We will have need to speak of synchronized $\Gamma$ subdivisons which are defined as follows:

DeFINITION 2. Synchronized $\Gamma$ subdivisions. Let $a<c<b$, so that $[a, c]$ and $[c, b]$ are adjoining closed intervals. Let

$$
\Gamma_{1}\left(x_{-1}, x_{0}, \ldots, x_{m}, x_{m+1}\right) \text { and } \Gamma_{2}\left(y_{-1}, y_{0}, \ldots, y_{n}, y_{n+1}\right)
$$

be, respectively, $\Gamma$ subdivisions of $[a, c]$ and $[c, b]$. If $x_{m-1}=y_{-1}$ and $x_{m+1}=y_{1}$, we will say that $\Gamma_{1}$ and $\Gamma_{2}$ are synchronized.

Definition 3. Refinements. We will say that $\Gamma_{1}$ is a refinement of $\Gamma_{2}$, and write $\Gamma_{1} \geqslant \Gamma_{2}$, if every point of $\Gamma_{2} \cap[a, b]$ beongs to $\Gamma_{1} \cap[a, b]$, and if $x_{-1}^{(1)}, x_{m+1}^{(1)} \in \Gamma_{1}$ and $x_{-1}^{(2)}, x_{n+1}^{(2)} \in \Gamma_{2}$ satisfy the conditions $a^{\prime} \leqslant x_{-1}^{(2)} \leqslant x_{-1}^{(1)}<a, b<x_{m+1}^{(1)} \leqslant x_{n+1}^{(2)} \leqslant b^{\prime}$.

Definition 4. The integral. Consider a $\Gamma\left(x_{-1}, x_{0}, \ldots, x_{n}, x_{n+1}\right)$ subdivision of $[a, b]$, and suppose that $f$ and $g$ are functions defined on $\left[a^{\prime}, b^{\prime}\right]$. The integral $(\sigma) \int_{a}^{b} f\left(d^{2} g / d x\right)$ is the real number $L$, if it exists uniquely, such that for each $\varepsilon>0$ there is a $\Gamma_{s}$ subdivision with the property: whenever $\Gamma \geqslant \Gamma_{\varepsilon}$ and $x_{i-1} \leqslant \xi_{i} \leqslant x_{i+1}$ for $i=1,2, \ldots, n-1$, then

$$
\begin{aligned}
\mid L-\left\{\frac{1}{2} f(a) V_{2}\left(g ; x_{-1}, x_{0}, x_{1}\right)\right. & +\sum_{i=1}^{n-1} f\left(\xi_{i}\right) V_{2}\left(g ; x_{i-1}, x_{i}, x_{i+1}\right) \\
& \left.+\frac{1}{2} f(b) V_{2}\left(g ; x_{n-1}, x_{n}, x_{n+1}\right)\right\} \mid<\varepsilon
\end{aligned}
$$

whenever $\Gamma \geqslant \Gamma_{\varepsilon}$.

For convenience, we shall often write the triple $\left\{x_{i-1}, x_{i}, x_{i+1}\right\}$ as $T_{i}$, and write the approximating sums for the integral as

$$
\sum_{\Gamma} f V_{2}(g ; T) \text { or } \sum_{i=0}^{n} f V_{2}\left(g ; T_{i}\right)
$$

where

$$
V_{2}\left(g ; T_{i}\right)=\delta_{i}\left[\frac{g\left(x_{i+1}\right)-g\left(x_{i}\right)}{x_{i+1}-x_{i}} \frac{g\left(x_{i}\right)-g\left(x_{i-1}\right)}{x_{i}-x_{i-1}}\right],
$$

and where $\delta_{i}=+1$ when $i=1, \ldots, n-1$, and $\delta_{i}=\frac{1}{2}$ when $i=0$ and $n$.

REMARK. If the integral exists, it is clear from Definitions 3 and 4 that it is independent of $a^{\prime}$ and $b^{\prime}$. 
DEFINITION 5. We define the oscillation function $\omega f V_{2}(g ;[a, b])$ to be equal to

$$
\sup _{\Gamma_{1}, \Gamma_{2}}\left|\sum_{\Gamma_{1}} f V_{2}(g ; T)-\sum_{\Gamma_{2}} f V_{2}(g ; T)\right|,
$$

where the supremum is taken over all $\Gamma_{1}$ and $\Gamma_{2}$ subdivisions of $[a, b]$ and the associated $\xi_{i}$ 's as in Definition 4.

Finally, for convenience, we include the well-known definition of oscillation of a function over an interval.

Definition 6. The oscillation of $f$ on a closed interval $I=[a, b]$ is defined to be

$$
\operatorname{osc}(f ; I)=\sup _{x, y \in I}|f(x)-f(y)| \text {. }
$$

\section{Necessary and sufficient conditions for integrability}

We begin with a Cauchy-type necessary and sufficient condition.

THEOREM 1. A necessary and sufficient condition that $(\sigma) \int_{a}^{b} f\left(d^{2} g / d x\right)$ exists is that for each $\varepsilon>0$ there is a $\Gamma_{8}$ subdivision of $[a, b]$ such that whenever $\Gamma_{1} \geqslant \Gamma_{\varepsilon}$ and $\Gamma_{2} \geqslant \Gamma_{\varepsilon}$,

$$
\left|\sum_{\Gamma_{1}} f V_{2}(g ; T)-\sum_{\Gamma_{2}} f V_{2}(g ; T)\right|<\varepsilon .
$$

PROOF. The necessity of the condition follows in the usual way.

For the sufficiency, we assume that for each $\varepsilon>0$ there exists a $\Gamma_{\varepsilon}$ subdivision such that

$$
\left|\sum_{\Gamma_{1}} f V_{2}(g ; T)-\sum_{\Gamma_{2}} f V_{2}(g ; T)\right|<\varepsilon
$$

whenever $\Gamma_{1} \geqslant \Gamma_{s}$ and $\Gamma_{2} \geqslant \Gamma_{\varepsilon}$. We construct a sequence $\left\{\Gamma_{n}\right\}$ of subdivisions such that $\Gamma_{n} \geqslant \Gamma_{n-1}$, and whenever $\Gamma^{\prime} \geqslant \Gamma_{n}$ and $\Gamma^{\prime \prime} \geqslant \Gamma_{n}$,

$$
\left|\sum_{\Gamma^{\prime}} f V_{2}(g ; T)-\sum_{\Gamma^{*}} f V_{2}(g ; T)\right|<\frac{1}{n} \text {. }
$$

Hence,

$$
\left|\sum_{\Gamma_{n+1}} f V_{2}(g ; T)-\sum_{\Gamma_{n+m}} f V_{2}(g ; T)\right|<\frac{1}{n}
$$

for all $m$ and $n$. Consequently $\left\{\Sigma_{\Gamma_{n}} f V_{2}(g ; T)\right\}$ is a Cauchy sequence of real numbers, and so has a limit $L$, say. Hence, for each $\varepsilon>0$, there exists $N(\varepsilon)$ such that

$$
\left|\sum_{\Gamma_{n}} f V_{2}(g ; T)-L\right|<\varepsilon \quad \text { whenever } n>N(\varepsilon)
$$


Furthermore,

$$
\left|\sum_{\Gamma_{n}} f V_{2}(g ; T)-\sum_{\Gamma} f V_{2}(g ; T)\right|<\varepsilon
$$

whenever $n-1>\varepsilon^{-1}$ and $\Gamma \geqslant \Gamma_{n-1}$. If we now choose $n>\max \left[N(\varepsilon), 1+\varepsilon^{-1}\right]$, and define $\Gamma_{\varepsilon}=\Gamma_{n-1}$, then it follows that $\left|\Sigma_{\Gamma} f V_{2}(g ; T)-L\right|<2 \varepsilon$ whenever $\Gamma \geqslant \Gamma_{\varepsilon}$. This concludes the proof.

REMARK. Each summation in (2) is of course multi-valued because of the choice of $\xi_{i}$ in Definition 4. The proof, however, remains valid for all such choices of $\xi_{i}$.

LEMMA 1. Let $\Gamma_{1}$ and $\Gamma_{2}$ be two $\Gamma$ subdivisons of $[a, b]$ such that $\Gamma_{2} \geqslant \Gamma_{1}$. Then

$$
\left|\sum_{\Gamma_{1}} f V_{2}(g ; T)-\sum_{\Gamma_{2}} f V_{2}(g ; T)\right| \leqslant \sum_{i=1}^{n} \omega f V_{2}\left(g ;\left[x_{i-1}, x_{i}\right]\right),
$$

where the $x_{i} \in \Gamma_{1}$.

Proof. To keep the details simple we consider a particular $\Gamma_{1}$ subdivision. The particular case will exhibit all properties of the general case. Accordingly, let $\Gamma_{1}$ be the subdivision $x_{-1}, x_{0}, x_{1}, x_{2}, x_{3}$, where $x_{-1}<a=x_{0}<x_{1}<x_{2}=b<x_{3}$. Let $\Gamma_{2} \geqslant \Gamma_{1}$ be obtained by inserting $l$ and $m$ extra points of subdivision in $\left(x_{0}, x_{1}\right)$ and $\left(x_{1}, x_{2}\right)$ respectively. Hence $\Gamma_{2}$ consists of points $y_{-1}, y_{0}, \ldots, y_{l+m+3}$, where

$$
y_{-1}<y_{0}=a<y_{1}<\ldots<y_{l+1}=x_{1}<\ldots<y_{l+m+2}=x_{2}<y_{l+m+3}=x_{3} .
$$

Then, suppressing the arguments of $f$ for convenience, we have

$$
\begin{aligned}
\sum_{\Gamma_{1}} f V_{2}(g ; T) & -\sum_{\Gamma_{2}} f V_{2}(g ; T) \\
= & \frac{1}{2} f V_{2}\left(g ; x_{-1}, x_{0}, x_{1}\right)+f V_{2}\left(g ; x_{0}, x_{1}, x_{2}\right)+\frac{1}{2} f V_{2}\left(g ; x_{1}, x_{2}, x_{3}\right) \\
& -\frac{1}{2} f V_{2}\left(g ; y_{-1}, y_{0}, y_{1}\right)-\sum_{i=1}^{l+m+1} f V_{2}\left(g ; y_{i-1}, y_{i}, y_{i+1}\right) \\
& -\frac{1}{2} f V_{2}\left(g ; y_{l+m+1}, y_{l+m+2}, y_{l+m+3}\right) \\
= & {\left[\frac{1}{2} f V_{2}\left(g ; x_{-1}, x_{0}, x_{1}\right)+\frac{1}{2} f V_{2}\left(g ; x_{0}, x_{1}, x_{2}\right)-\frac{1}{2} f V_{2}\left(g ; y_{-1}, y_{0}, y_{1}\right)\right.} \\
& \left.\quad-\sum_{i=1}^{l} f V_{2}\left(g ; y_{i-1}, y_{i}, y_{i+1}\right)-\frac{1}{2} f V_{2}\left(g ; y_{l}, y_{l+1}, y_{l+2}\right)\right] \\
& +\left[\frac{1}{2} f V_{2}\left(g ; x_{0}, x_{1}, x_{2}\right)+\frac{1}{2} f V_{2}\left(g ; x_{1}, x_{2}, x_{3}\right)-\frac{1}{2} f V_{2}\left(g ; y_{l}, y_{l+1}, y_{l+2}\right)\right. \\
& \left.\quad-\sum_{i=l+2}^{l+m+1} f V_{2}\left(g ; y_{i-1}, y_{i}, y_{i+1}\right)-\frac{1}{2} f V_{2}\left(g ; y_{l+m+1}, y_{l+m+2}, y_{l+m+3}\right)\right] \\
= & {\left[\sum_{\Gamma_{1}^{\prime}} f V_{2}(g ; T)-\sum_{\Gamma_{2}^{\prime}} f V_{2}(g ; T)\right]+\left[\sum_{\Gamma_{1}^{\prime}} f V_{2}(g ; T)-\sum_{\Gamma_{2}^{*}} f V_{2}(g ; T)\right], }
\end{aligned}
$$


where $\Gamma_{1}^{\prime}$ and $\Gamma_{2}^{\prime}$ are $\Gamma$ subdivisions of $\left[x_{0}, x_{1}\right]$ and $\Gamma_{1}^{\prime \prime}$ and $\Gamma_{2}^{\prime \prime}$ are $\Gamma$ subdivisions of $\left[x_{1}, x_{2}\right]$. Hence,

$$
\left|\sum_{\Gamma_{1}} f V_{2}(g ; T)-\sum_{\Gamma_{2}} f V_{2}(g ; T)\right| \leqslant \sum_{i=1}^{2} \omega f V_{2}\left(g ;\left[x_{i-1}, x_{i}\right]\right) .
$$

The extension of this result to $\Gamma_{1}$ subdivisions containing more than five points is straightforward and, as indicated earlier, the details will be omitted.

THEOREM 2. A necessary and sufficient condition that $(\sigma) \int_{a}^{b} f\left(d^{2} g / d x\right)$ exists is that

$$
\inf _{\Gamma} \sum_{\Gamma} \omega f V_{2}(g ; I) \equiv \inf _{\Gamma} \sum_{i=1}^{n} \omega f V_{2}\left(g ;\left[x_{i-1}, x_{i}\right]\right)=0 .
$$

PRoof. We first show that the condition is sufficient. Accordingly, suppose that (3) holds. Then, for each $\varepsilon>0$ there exists a $\Gamma_{\varepsilon}$ subdivision such that

$$
\sum_{\Gamma \varepsilon} \omega f V_{2}(g ; I)<\varepsilon \text {. }
$$

Now suppose that $\Gamma_{1} \geqslant \Gamma_{\varepsilon}$. Then, using Lemma 1, we obtain

$$
\left|\sum_{\Gamma_{\varepsilon}} f V_{2}(g ; T)-\sum_{\Gamma_{1}} f V_{2}(g ; T)\right| \leqslant \sum_{\Gamma_{\varepsilon}} \omega f V_{2}(g ; I)<\varepsilon .
$$

The existence of the integral now follows from Theorem 1.

To prove the condition necessary we assume that for each $\varepsilon>0$ there exists $\Gamma_{\text {s }}$ such that whenever $\Gamma_{1} \geqslant \Gamma_{\varepsilon}$ and $\Gamma_{2} \geqslant \Gamma_{e}$,

$$
\left|\sum_{\Gamma_{1}} f V_{2}(g ; T)-\sum_{\Gamma_{2}} f V_{2}(g ; T)\right|<\varepsilon .
$$

Let $\Gamma \geqslant \Gamma_{\varepsilon}$ and let $\Gamma$ consist of the points $x_{-1}, x_{0}, \ldots, x_{n}, x_{n+1}$. For each subinterval $\left[x_{i-1}, x_{i}\right], i=1,2, \ldots, n$, Definition 5 shows that we can find subdivisions $\Gamma_{i}^{\prime}$ and $\Gamma_{i}^{\prime \prime}$ of $\left[x_{i-1}, x_{i}\right]$ such that

$$
\left|\sum_{\Gamma_{i}^{\prime}} f V_{2}(g ; T)-\sum_{\Gamma_{i}^{\prime}} f V_{2}(g ; T)\right|>\omega f V_{2}\left(g ;\left[x_{i-1}, x_{i}\right]\right)-\frac{\varepsilon}{n} .
$$

By interchanging $\Gamma_{i}^{\prime}$ and $\Gamma_{i}^{\prime \prime}$ if necessary we can also have

$$
\sum_{\Gamma_{i}} f V_{2}(g ; T)-\sum_{\Gamma_{i}^{*}} f V_{2}(g ; T) \geqslant 0,
$$

and the modulus signs in the previous inequality can be omitted. If we put $\Gamma^{\prime}=\bigcup_{i=1}^{n} \Gamma_{i}^{\prime}$ and $\Gamma^{\prime \prime}=\bigcup_{i=1}^{n} \Gamma_{i}^{\prime \prime}$, then $\Gamma^{\prime} \geqslant \Gamma_{s}, \Gamma^{\prime \prime} \geqslant \Gamma_{e}$, and

$$
0 \leqslant \sum_{i=1}^{n} \omega f V_{2}\left(g ;\left[x_{i-1}, x_{i}\right]\right) \leqslant \sum_{i=1}^{n}\left[\sum_{\Gamma^{\prime}} f V_{2}(g ; T)-\sum_{\Gamma_{i}^{\prime \prime}} f V_{2}(g ; T)\right]+\varepsilon
$$

$$
<\varepsilon+\varepsilon=2 \varepsilon
$$


provided that $\Gamma_{1}^{\prime}, \ldots, \Gamma_{n}^{\prime}$ are synchronized, and $\Gamma_{1}^{\prime \prime}, \ldots, \Gamma_{n}^{\prime \prime}$ are also synchronized. When the subdivisions are not synchronized we can make use of (5) and this will have the effect of introducing an extra $\varepsilon$ in (6). The required result now follows.

COROLLARY. If $(\sigma) \int_{a}^{b} f\left(d^{2} g / d x\right)$ exists, then

$$
\inf _{\Gamma}^{n-1} \operatorname{osc}\left(f ;\left[x_{i-1}, x_{i+1}\right]\right)\left|V_{2}\left(g ; T_{i}\right)\right|=0 .
$$

Proof. Let $\Gamma_{1}$ and $\Gamma_{2}$ be identical $\Gamma$ subdivisions of an interval $[c, d]$. Denote their points by $x_{-1}, x_{0}, x_{1}, x_{2}, x_{3}$, where $x_{-1}<x_{0}=c<x_{1}<x_{2}=d<x_{3}$. Since $\xi_{i}$ in Definition 4 is arbitrary within the subinterval $\left[x_{i-1}, x_{i+1}\right]$, we choose $\xi_{1}=\alpha$ and $\xi_{1}=\beta$, respectively for the $\Gamma_{1}$ and $\Gamma_{2}$ subdivisions. It then follows from Definition 5 that

$$
\omega f V_{2}(g ;[c, d]) \geqslant|f(\alpha)-f(\beta)|\left|V_{2}\left(g ; x_{0}, x_{1}, x_{2}\right)\right|
$$

whenever $\alpha$ and $\beta$ are in $[c, d]$. Hence, replacing $[c, d]$ by $\left[x_{i-1}, x_{i+1}\right]$ and making other obvious changes, we have

$$
\omega f V_{2}\left(g ;\left[x_{i-1}, x_{i+1}\right)\right) \geqslant \operatorname{osc}\left(f ;\left[x_{i-1}, x_{i+1}\right]\right)\left|V_{2}\left(g ; x_{i-1}, x_{i}, x_{i+1}\right)\right| .
$$

The required result now follows readily from Theorem 2 .

The following discussion motivates the next theorem. Consider the function $g$ defined by

$$
\begin{aligned}
& g(x)=\beta x, \quad x \geqslant 0, \\
& g(x)=\alpha x, \quad x \leqslant 0,
\end{aligned}
$$

where $\alpha$ and $\beta$ are constants. Consider a $\Gamma$ subdivision of $[-1,1]$, and let $0=x_{p} \in \Gamma$. Then, if $f(x) \equiv 1$,

$$
\sum_{\Gamma} f V_{2}(g ; T)=(\beta-\alpha) f\left(\xi_{p}\right),
$$

where $x_{p-1} \leqslant \xi_{p} \leqslant x_{p+1}$. Consequently, if $(\sigma) \int_{-1}^{1} f\left(d^{2} g / d x\right)$ exists, it must have the value $(\beta-\alpha) f(0)$. Hence, if $f$ is discontinuous at 0 , we must have $\beta=\alpha$, in which case $g$ is differentiable at 0 . On the other hand, if $\alpha=g_{-}^{\prime}(0) \neq \beta=g_{+}^{\prime}(0)$, then $f$ must be continuous at 0 .

TheOREM 3. If $(\sigma) \int_{a}^{b} f\left(d^{2} g / d x\right)$ exists, and $a<c<b$, then the conditions $f$ discontinuous at $c$, and $g$ non-differentiable at $c$ cannot occur simultaneously. 
Proof. If $(\sigma) \int_{a}^{b} f\left(d^{2} g / d x\right)$ exists, then it follows from Theorem 2, Corollary, that for each $\varepsilon>0$ there exists $\Gamma_{s}$ such that whenever $\Gamma \geqslant \Gamma_{s}$,

$$
\sum_{i=1}^{n-1} \sup _{\xi_{i}, \eta_{i} \in I_{i}}\left|f\left(\xi_{i}\right)-f\left(\eta_{i}\right)\right|\left|V_{2}\left(g ; x_{i-1}, x_{i}, x_{i+1}\right)\right|<\varepsilon,
$$

where $I_{i}=\left[x_{i-1}, x_{i+1}\right]$.

If $c$ is a point of discontinuity of $f$, then by including $c$ in $\Gamma$ it follows that if $c=x_{p}$,

where

$$
\sup _{x, y \in I_{p}}|f(x)-f(y)|\left|Q_{1}\left(g ; x_{p+1}, c\right)-Q_{1}\left(c, x_{p-1}\right)\right|<\varepsilon,
$$

$$
Q_{1}(g ; x, y)=\frac{g(y)-g(x)}{y-x} .
$$

Since $c$ is a point of discontinuity of $f$, there exists a positive number $k$ such that

$$
\sup _{x, y \in I_{p}}|f(x)-f(y)|>k
$$

no matter how small $x_{p+1}-x_{p-1}$. Consequently, no matter how small $x_{p+1}-x_{p-1}$,

$$
\left|Q_{1}\left(g ; x_{p+1}, c\right)-Q_{1}\left(g ; c, x_{p-1}\right)\right|<\varepsilon / k .
$$

Since $x_{p-1}$ and $x_{p+1}$ are independent, it follows from Cauchy's principle of convergence that $g_{-}^{\prime}(c)$ and $g_{+}^{\prime}(c)$ both exist, and are equal. Thus, we have shown that if $f$ is discontinuous at $c$, then $g$ must be differentiable at that point. It now follows that if $g$ is not differentiable at $c$, then $f$ must be continuous there. This completes the proof of the theorem.

CoRollary. If $(\sigma) \int_{a}^{b} f\left(d^{2} g / d x\right)$ exists, then the conditions $f$ discontinuous and $g$ non-differentiable on the right at a cannot occur simultaneously. Similarly $f$ discontinuous and $g$ non-differentiable on the left at $b$ cannot occur simultaneously.

\section{An application}

THEOREM 4. If $a<c<b$, and any two of the integrals

$$
\begin{aligned}
& \text { (o) } \int_{a}^{c} f \frac{d^{2} g}{d x}, \\
& \text { ( } \sigma) \int_{c}^{b} f \frac{d^{2} g}{d x} \text { and } \\
& \text { (o) } \int_{a}^{b} f \frac{d^{2} g}{d x}
\end{aligned}
$$

exist, then so does the other, and

$$
(\sigma) \int_{a}^{b} f \frac{d^{2} g}{d x}=(\sigma) \int_{a}^{c} f \frac{d^{2} g}{d x}+(\sigma) \int_{c}^{b} f \frac{d^{2} g}{d x} .
$$


ProOF. We shall only prove one case, the proofs of other cases being similar to the one given.

Accordingly, assume that $(\sigma) \int_{a}^{b} f\left(d^{2} g / d x\right)$ exists. Then it follows from Theorem 2 that the other two integrals in (7) also exist. Consequently, given $\varepsilon>0$ there exist subdivisions $\Gamma_{\mathrm{s}}^{\prime}$ and $\Gamma_{\mathrm{s}}^{\prime \prime}$ of $[a, c]$ and $[c, b]$ such that

$$
\left|\sum_{\Gamma^{\prime}} f V_{2}(g ; T)-L^{\prime}\right|<\frac{1}{2} \varepsilon \quad \text { whenever } \Gamma^{\prime} \geqslant \Gamma_{\varepsilon}^{\prime}
$$

and

$$
\left|\sum_{\Gamma^{\prime \prime}} f V_{2}(g ; T)-L^{\prime \prime}\right|<\frac{1}{2} \varepsilon \quad \text { whenever } \Gamma^{\prime \prime} \geqslant \Gamma_{s^{\prime \prime}}^{\prime \prime}
$$

where

$$
L^{\prime}=(\sigma) \int_{a}^{c} f \frac{d^{2} g}{d x} \text { and } L^{\prime \prime}=(\sigma) \int_{c}^{b} f \frac{d^{2} g}{d x} .
$$

Let $\Gamma_{e}^{\prime}$ consist of the points $x_{-1}, x_{0}=a, x_{1}, \ldots, x_{m}=c, x_{m+1}$, and let $\Gamma_{\varepsilon}^{\prime \prime}$ consist of the points $y_{-1}, y_{0}=c, y_{1}, \ldots, y_{n}=b, y_{n+1}$. If the subdivisions $\Gamma_{\varepsilon}^{\prime}$ and $\Gamma_{e}^{\prime \prime}$ are not synchronized, several cases can arise. One of these will be considered; others can be dealt with in a similar way. Consequently, suppose that

$$
y_{-1}<x_{m-1}<x_{m}=c=y_{0}<x_{m+1}<y_{1} .
$$

Let $\Gamma_{s}^{*}$ be the refinement of $\Gamma_{s}^{\prime \prime}$ obtained by choosing $y_{-1}^{*}=x_{m-1}$ and introducing an additional point $y_{1}^{*}=x_{m+1}$. Then $\Gamma_{*}^{*}$ consists of the points $y_{-1}^{*}=x_{m-1}$, $y_{0}=x_{m}, y_{1}^{*}=x_{m+1}, y_{1}, \ldots, y_{n}=b, y_{n+1}$. Then

$$
\left|\sum_{\Gamma^{\prime \prime}} f V_{2}(g ; T)-L^{\prime \prime}\right|<\frac{1}{2} \varepsilon \quad \text { whenever } \Gamma^{\prime \prime}>\Gamma_{s}^{*},
$$

and from (8)

$$
\left|\sum_{\Gamma^{\prime}} f V_{2}(g ; T)-L^{\prime}\right|<\frac{1}{2} \varepsilon \quad \text { whenever } \Gamma^{\prime} \geqslant \Gamma_{\varepsilon^{\prime}}^{\prime}
$$

We observe that $\Gamma_{s}^{\prime}$ and $\Gamma_{s}^{*}$ are now synchronized. Consequently there is no loss of generality in assuming that (8) and (9) are valid for synchronized subdivisions $\Gamma_{\text {s }}^{\prime}$ and $\Gamma_{8^{\prime \prime}}$.

Let $\Gamma_{s}=\Gamma_{s}^{\prime} \cup \Gamma_{s}^{\prime \prime}$, and let $\Gamma$ be any subdivision of $[a, b]$ such that $\Gamma \geqslant \Gamma_{\varepsilon}$. Then we can write $\Gamma=\Gamma^{\prime} \cup \Gamma^{\prime \prime}$, where by the above discussion, $\Gamma^{\prime}$ and $\Gamma^{\prime \prime}$ are synchronized subdivisions of $[a, c]$ and $[c, b]$ respectively. If $\Gamma^{\prime}$ and $\Gamma^{\prime \prime}$ consist, respectively, of the points

$$
x_{-1}, \quad x_{0}=a, \ldots, x_{m}=c, \quad x_{m+1}
$$

and

$$
x_{m-1}, \quad x_{m}=c, \quad x_{m+1}, \ldots, x_{m+n}=b, \quad x_{m+n+1},
$$


then

$$
\begin{aligned}
\sum_{\Gamma} f V_{2}(g ; T)=\{ & \left\{\frac{1}{2} f(a) V_{2}\left(g ; T_{0}\right)+\sum_{i=1}^{m-1} f\left(\xi_{i}\right) V_{2}\left(g ; T_{i}\right)+\frac{1}{2} f(c) V_{2}\left(g ; T_{m}\right)\right\} \\
& +\left\{\frac{1}{2} f(c) V_{2}\left(g ; T_{m}\right)+\sum_{i=m+1}^{m+n-1} f\left(\xi_{i}\right) V_{2}\left(g ; T_{i}\right)+\frac{1}{2} f(b) V_{2}\left(g ; T_{m+n}\right)\right\} \\
& +\left\{f\left(\xi_{m}\right)-f(c)\right\} V_{2}\left(g ; T_{m}\right) .
\end{aligned}
$$

It now follows from the proof of Theorem 3 that the last term in (10) tends to zero under refinement irrespective of whether $f$ is continuous or discontinuous at $c$. Hence, from (10), the limit of $\Sigma_{\Gamma} f V_{2}(g ; T)$ under refinement exists by assumption and equals $L^{\prime}+L^{\prime \prime}$, as required.

I would like to express my appreciation to Professor E. R. Love for several helpful comments and suggestions relating to the preparation of this paper.

\section{References}

T. H. Hildebrandt (1963), Introduction to the theory of integration (Pure and Appl. Math. Vol. 13, Academic Press, New York).

A. M. Russell (1973), 'Functions of bounded $k$ th variation, and Stieltjes-type integrals' (Doctoral dissertation, University of Melbourne).

A. M. Russell (1975), 'Stieltjes-type integrals', J. Austral. Math. Soc. (Ser A) 20, 431-448.

Department of Mathematics

University of Melbourne

Parkville 3052

Australia 